\title{
SQUARE SUMMABILITY OF VARIATIONS OF $g$-FUNCTIONS AND UNIQUENESS OF $g$-MEASURES
}

\author{
ANDERs JOHANSSON AND ANDERs ÖBERG
}

\begin{abstract}
We prove uniqueness of $g$-measures for $g$-functions satisfying quadratic summability of variations. Our result is in contrast to the situation of, e.g., the one-dimensional Ising model with long-range interactions, since $\ell_{1}$-summability of variations is required for general potentials. We illustrate this difference with some examples. To prove our main result we use a product martingale argument. We also give conditions for uniqueness of general $G$-measures, i.e., the case for general potentials, based on our investigation of the probabilistic case involving $g$-functions.
\end{abstract}

\section{Introduction}

In [8] Keane introduced the concept of $g$-measures, but the origin of "chains with complete connections" goes back to (at least) Doeblin and Fortet [4]. For a survey of the history and prehistory of some important results concerning $g$ measures and related results, we refer to the recent [14]. Here we are concerned with the problem of uniqueness of $g$-measures.

Let $T$ be an $m$-to-1 $(m \geq 2)$ covering transformation of a compact metric space $(X, d)$. Let $G$ be a positive measurable function $G: X \rightarrow \mathbb{R}_{+}$. If

$$
\sum_{y \in T^{-1} x} G(y)=1, \quad \text { for all } x \in X
$$

then we call $G$ a $g$-function. A $G$-measure is a $T$-invariant Borel probability measure which is an eigen-measure corresponding to the maximal eigenvalue of the adjoint $\mathcal{L}_{G}^{*}$ of the transfer operator

$$
\mathcal{L}_{G} f(x)=\sum_{y \in T^{-1} x} G(y) f(y), \quad f \in C(X) .
$$

Such a $\mu$ always exists if $G$ is a continuous function (this follows from the Schauder-Tychonoff fixed point theorem).

In this paper we assume the existence of an eigen-measure $\mu$ and investigate conditions on $G$ that imply uniqueness of $G$-measures. Our main result is really about a class of iterated function systems (including "overlapping" systems) where the concept of a $g$-function corresponds to what we call probabilistic

Received September 29, 2002.

2000 Mathematics Subject Classification. 28D05, 37A30, 37C30.

Key words and phrases. $g$-function, $g$-measure, Ising model with long-range interactions. 
weights. But, in this introductory section, we suppose that $(T, X)$ is a dynamical system on a compact metric space $(X, d)$ in order to make the connection with $g$-functions and $g$-measures.

We will moreover assume that $T$ can be associated to an iterated function system with continuous functions on a compact metric space in the standard way: By choosing $m$ inverse branches of $T$ and possibly in conjunction with a topological cut of the space $X$. We will not deal with the issue when this association with an IFS is possible, we think it is enough to note that the canonical example of a covering transformation, the symbolic shift, is well-behaved in this regard. By a symbolic shift we mean the left shift on the space $X=\Sigma^{\mathbb{Z}_{+}}$ of sequences $x=\left(x_{i}\right)_{i=1}^{\infty}, x_{i} \in \Sigma$, where $\Sigma$ is a finite set of $m$ symbols. We will assume the metric $d\left(x, x^{\prime}\right)=2^{-\min \left\{t \mid x_{t} \neq x_{t}^{\prime}\right\}}$. The disconnected structure of the symbolic shift space ensures that the $m$ inverse branches of the shift are continuously defined.

For a well-behaved system, the images of the inverse branches of $T^{n}$ partition $X$ into $m^{n}$ inverse images. It is enough that these sets are disjoint $\bmod \mu$ for all $g$-measures $\mu$. Let $I_{-n}(x)$ denote the a.s. unique $n$th inverse image containing $x$ and define the $n$th variation of $g$ by

$$
\operatorname{var}_{n} g(x)=\sup _{y, z \in I_{-n}(x)}|g(y)-g(z)| .
$$

Our main result, Theorem 3.1, can now be stated as follows in this context.

Theorem 1.1. Assume that $g$ is a $g$-function, $\inf g>0$, and that $\mu$ is a $g$ measure such that diam $I_{-n} \rightarrow 0$, $\mu$-almost surely. Suppose that $\left(R_{n}\right)_{n=1}^{\infty}$ is a (stochastic) sequence, such that each $R_{n}$ is measurable with respect to the algebra generated by $I_{-n}$ and that $\sum_{n} R_{n}^{2} \in L^{1}(\mu)$ and suppose that

$$
\sum_{n} \mu\left\{\operatorname{var}_{n} g(x)>R_{n}\right\}<\infty
$$

then we have a unique g-measure.

Corollary 1.2. We have at most one $g$-measure if $\operatorname{diam} I_{-n} \rightarrow 0$ and if

$$
\sum_{n=1}^{\infty} r_{n}^{2}<\infty
$$

where $r_{n}=\sup _{x} \operatorname{var}_{n} g(x)$.

Remark 1. Observe that since inf $g>0$, we may use $\operatorname{var}_{n} \log g$ instead of $\operatorname{var}_{n} g$ in the square summability conditions of Theorem 1.1 and Corollary 1.2. Conditions on $\operatorname{var}_{n} \log g$ are standard in the literature, see, e.g., [15].

Remark 2. Note that if we take $R_{n}:=\operatorname{var}_{n} g$ in Theorem 1.1, then the condition becomes that $\sum_{n}\left(\operatorname{var}_{n} g(x)\right)^{2}$ should be integrable with respect to some $g$-measure $\mu$.

Remark 3 . We will sometimes refer to a condition like (1.2) as $\ell_{2}$-summability, and in general $\ell_{p}$-summability for other exponents. 
The following example illustrates an important difference between one-sided and two-sided "spin systems". Compare with Example 3 below.

Example 1. Take $T$ to be the shift-operator on $\{+1,-1\}^{\mathbb{Z}_{+}}$and define the $g$-function

$$
g\left( \pm 1, x_{1}, x_{2}, \ldots\right)=\exp ( \pm B(x)) / 2 \cosh (B(x))
$$

where, for $\beta>0, \alpha>1$,

$$
B(x)=B\left(x_{1}, x_{2}, \ldots\right)=\beta \cdot \sum_{i=1}^{\infty} \frac{x_{i}}{i^{\alpha}} .
$$

It is then readily checked that $\operatorname{var}_{n} g=O\left(n^{-\alpha+1}\right)$ and that Theorem 1.1 implies the uniqueness of $g$-measures if $\alpha>3 / 2$. The existence follows from the fact that $g$ is continuous.

Bramson and Kalikow [3] constructed an example showing that continuity and "regularity" (inf $g>0$ ) of the $g$-function is not enough for uniqueness. Later Quas [13] constructed an example for an expanding $C^{1}$ covering map on the circle, using ideas from [3].

Our proof does not depend on the coupling method, as in [2] for example. Instead we rely on a classic product martingale idea of Kakutani. We show uniqueness by proving uniform integrability relative $\mu$ of

$$
M_{-n}(x):=\tilde{\mu}\left\{I_{-n}(x)\right\} / \mu\left\{I_{-n}(x)\right\}, n \geq 0,
$$

where $\mu$ and $\tilde{\mu}$ are two hypothetical $g$-measures. Then we can take the relation (1.1) into account in ways that the standard coupling argument does not allow us to do.

We do not know if the square summability condition is optimal in the sense that $\ell_{p}$-summability is not enough for uniqueness when $p>2$. We think that it is an interesting problem to decide whether or not such a best $p$ exists. The example by Bramson and Kalikow does not, at least explicitly, give a bound for such a $p$. The Ising model described in Example 3 below shows that for general weights $G$ the condition of $\ell_{1}$-summability of variations is sharp in the sense that we do not necessarily have uniqueness for $\ell_{p}$-summability, $p>1$. We believe that it would be particularly interesting to find a critical value in a simple model similar to the one given above in Example 1.

For $g$-functions, it is known that there are weaker conditions than summability of variations that produce uniqueness of $g$-measures. In [14] we find a survey of some results in the past. Already in 1955, Harris [7] discovered quite a weak condition. Here we compare our result with a closely related condition investigated by Berbee [2]. Berbee considered symbolic shift spaces and the condition

$$
\sum_{n=1}^{\infty} \exp \left\{-\sum_{i=1}^{n} r_{i}\right\}=\infty
$$


where $r_{i}=\sup _{x} \operatorname{var}_{i} \log g(x)$. This is clearly a weaker condition than $\ell_{1}$-summability and not comparable with the condition of square summability $\sum_{1}^{\infty} r_{n}^{2}<$ $\infty$.

Example 2. It is easy to produce sequences $r_{n} \in \ell_{2}$ that do not satisfy (1.4): just take any $r_{n} \asymp n^{-\alpha}, 1 / 2<\alpha<1$.

To see that condition $r_{n} \in \ell_{2}$ is not weaker than (1.4): Let $r_{n}, n \geq 1$, take values $\epsilon_{k}, k \geq 1$, for blocks $M_{k} \leq n<M_{k+1}$ chosen such that the lengths $M_{k+1}-M_{k}=\epsilon_{k}^{-2}$ where $\epsilon_{k}$ hence is chosen such that $\epsilon_{k}^{-2}$ is a positive integer. In this way, summing the positive series $\sum_{n} r_{n}^{2}$ block-wise gives

$$
\sum_{k}\left(M_{k+1}-M_{k}\right) \cdot \epsilon_{k}^{2}=\sum_{k} 1 .
$$

The condition of square summability is then clearly not satisfied. The Berbee condition (1.4) can however be satisfied for a suitable choice of $\epsilon_{k}$. The blockwise partial sums of (1.4)

$$
S_{k}:=\sum_{n=M_{k}}^{M_{k+1}-1} \exp \left\{-\sum_{i=1}^{n} r_{i}\right\}
$$

are geometric sums on $M_{k+1}-M_{k}$ terms with quotient $e^{-\epsilon_{k}}$ and initial term $\exp \left\{-\sum_{i=1}^{M_{k}-1} r_{i}\right\} \cdot e^{-\epsilon_{k}}$. Therefore,

$$
S_{k}=e^{-\sum_{l=1}^{k-1} \epsilon_{l}^{-1}} \cdot \frac{1-e^{-\epsilon_{k}^{-1}}}{e^{\epsilon_{k}}-1},
$$

since $\sum_{i=1}^{M_{k}-1} r_{i}$ equals $\sum_{l=1}^{k-1}\left(M_{l+1}-M_{l}\right) \cdot \epsilon_{l}=\sum_{l=1}^{k-1} \epsilon_{l}^{-1}$. The quotients $S_{k+1} / S_{k}$ of consecutive terms of the series in (1.4) summed block-wise are thus

$$
S_{k+1} / S_{k}=e^{-\epsilon_{k}^{-1}} \cdot \frac{1-e^{-\epsilon_{k+1}^{-1}}}{e^{\epsilon_{k+1}}-1} / \frac{1-e^{-\epsilon_{k}^{-1}}}{e^{\epsilon_{k}}-1} .
$$

Simple approximations, $e^{\epsilon_{k}}-1 \approx \epsilon_{k}$ and $1-e^{-\epsilon_{k}^{-1}} \approx 1$ for small $\epsilon_{k}$, shows that this is greater than $\frac{1}{2} \cdot e^{-\epsilon_{k}^{-1}} \cdot \frac{\epsilon_{k}}{\epsilon_{k+1}}$ for, say, $\epsilon_{k} \leq 1 / 10$. Hence, by letting $\epsilon_{1}=1 / 10$ and taking $\epsilon_{k+1} \leq \frac{1}{2} \cdot e^{-\epsilon_{k}^{-1}} \cdot \epsilon_{k}$ such that $\epsilon_{k+1}^{-2}$ is a positive integer, we obtain that $S_{k+1} / S_{k} \geq 1$. The series $\sum_{k} S_{k}$ in (1.4) will consequently diverge.

When one is concerned with general potentials, a standard condition is that of summable variation (or Dini continuity, see below) of $G$; see for instance [12], [11], [5] and [6]. In cases of proving uniqueness of a $G$-measure one usually uses the existence of a strictly positive eigen-function of the transfer operator. With a positive eigen-function $h$ established one may then define a $g$-function $g$ by

$$
g(x):=G(x) \cdot h(x) / \lambda \cdot h(T x),
$$

where $\lambda$ is the maximum eigenvalue of the transfer operator. Then $G$-measures $\nu$ and $g$-measures $\mu$ are in a one-to-one correspondence with $\mu=h \nu$. However, the construction of $h$ is usually carried out by some compactness argument and 
it seems to be hard, in general, to estimate the variations of $h$ and the resulting $g$.

Our next example shows, a little surprisingly, that square summability may not be stable under the normalization (1.5). This is in contrast to the situation of summability, since that condition is stable under the normalization (1.5).

Example 3. Consider Example 1, but now let

$$
G\left( \pm 1, x_{1}, x_{2} \ldots\right)=\exp ( \pm B(x))
$$

This is not a $g$-function since the weights now sum to $2 \cosh (B(x))$.

If one carries out the transformation above (provided that an eigen-function $h$ exists) the resulting measure $h \nu$ will give a variant of the one-dimensional Ising model with long-range interactions. It is known in this case, see [1], that $\alpha=2$ is the critical value for uniqueness of a corresponding $G$-measure. We have uniqueness when $\alpha>2$ and non-uniqueness for $\alpha \leq 2$ and suitably large $\beta$. Our result hence implies that the variations of $g(x)=G(x) h(x) / \lambda h(T x)$ are not square summable for $3 / 2<\alpha \leq 2$, although this is the case for the variations of $G$.

We have discussed summability conditions on $r_{n}=\sup _{x} \operatorname{var}_{n} G(x)$, i.e., we start from a uniform bound on the $n$th variations. A condition often used is Dini continuity. A function $G$ is Dini continuous if

$$
\sum_{n \geq 1} \Omega\left(G, \eta^{n}\right)<\infty
$$

for some (and then any) $\eta<1$, where $\Omega(G, t):=\sup _{d(x, y) \leq t}|G(x)-G(y)|$.

Our result covers the weaker condition

$$
\sum_{n \geq 1}\left(\Omega\left(G, \eta^{n}\right)\right)^{2}<\infty
$$

One should note that the conditions of Theorem 1.1 apply to situations when the uniform bound on $r_{n}=\sup _{x} \operatorname{var}_{n} g(x)$ does not apply. The conditions on $\operatorname{var}_{n} g(x)$ could, for instance, be verified for all Borel probability measures $\mu$ so that the theorem applies as soon as one has settled the question of existence of $g$-measures. Similar conditions and arguments is used by Keller in [10] where he considers piecewise monotonic systems $T: X \rightarrow X$ on an interval $X=[a, b] \subset \mathbb{R}$ with general weights $G(x)$ that has bounded (universal) p-variation, i.e.

$$
\sup \sum_{j=0}^{n-1}\left|G\left(t_{j+1}\right)-G\left(t_{j}\right)\right|^{p}<\infty, \quad p \geq 1,
$$

where the supremum is taken over all subdivisions $a=t_{0}<t_{1}<\cdots<t_{n}=b$ of the interval. It is not too hard to show that any $g$-function having bounded $p$ variation will satisfy the conditions of Theorem 1.1 for any probability measure $\mu$. The point we wish to make here is that one may find natural criteria on $g$ such that the theorem applies without imposing uniform bounds on $\operatorname{var}_{n} g(x)$. 
The rest of this paper is structured as follows: In Section 2 we give some preliminaries of iterated function systems. In Section 3 we state our main result, which is then proved in Section 4. In Section 5 we give a new way of looking at the case with general weights, i.e., when $\sum_{y: T y=x} G(y) \neq 1$.

\section{Iterated function systems}

We will work in another setting than the formalism of $g$-functions for covering transformations. We study iterated function systems which are represented as a dynamical shift system on sequences of symbols and points in a compact metric space. The reason for choosing the following formalism is that we want to allow for a more general class of iterated function systems than the non-overlapping ones which arise, e.g., from inverse branches of covering transformations.

Let $(X, d)$ be a compact metric space and $\Sigma$ a finite set of symbols. By an iterated function system $\mathfrak{S}$ (IFS) on $\Sigma \times X$ we mean a continuous map

$$
\Sigma \times X \ni(\sigma, x) \stackrel{\mathfrak{S}}{\longrightarrow} \sigma x \in X .
$$

Since $\Sigma$ is discrete, the IFS $\mathfrak{S}$ associates to each symbol $\sigma \in \Sigma$ a unique continuous map $X \rightarrow X$, which we will often refer to by the corresponding symbol $\sigma$. A word $\omega=\omega_{1} \omega_{2} \cdots \omega_{n} \in \Sigma^{n}$ will refer to the composition $\omega_{n} \circ \cdots \circ \omega_{1}$ of the corresponding maps.

We are interested in weighted IFS, i.e., pairs $(\mathfrak{S}, q)$ with a measurable nonnegative mapping

$$
\Sigma \times X \ni(\sigma, x) \stackrel{q}{\rightarrow} q(\sigma, x) \in[0, \infty) .
$$

such that, for any fixed $x \in X, q(\sigma, x) \neq 0$, for some $\sigma \in \Sigma$.

We will work with the case when we have probabilistic weights, i.e., we have a weight function $p$ such that $\sum_{\sigma} p(\sigma, x)=1$ for all $x \in X$. In this case we think of the weights as defining the transition probabilities of a Markov chain $\left(X_{n}\right)_{n \geq 0}$, on $X$, where $X_{n+1}$ is chosen among $\sigma X_{n}, \sigma \in \Sigma$ according to the probability $p\left(\sigma, X_{n}\right)$.

A stationary measure on $X$ for such a Markov chain $X_{n}, n \geq 0$, is a probability measure invariant under the action of the adjoint $\mathcal{L}_{p}^{*}$ of the transition operator (defined on measurable functions)

$$
\mathcal{L}_{p} f(x)=\sum_{\sigma} p(\sigma, x) f(\sigma x) .
$$

The natural extensions of the systems we are considering correspond to biinfinite Markov chains $\left(W_{n}, X_{n}\right)_{n \in \mathbb{Z}}$ on $\Sigma \times X$, such that $X_{n+1}=W_{n} X_{n}$ and such that the distribution of $W_{n} \in \Sigma$ conditioned on $X_{n}$ is given by the probabilities $p\left(\cdot, X_{n}\right)$. The distribution of $\left(X_{n}\right)$ is then a Markov chain on $X$ as before and the chain $\left(W_{n}, X_{n}\right)$ is merely a lifting of $\left(X_{n}\right)$ with the symbolic information retained. The sample space $\Omega$ of sequences $\boldsymbol{\omega}=\left(\omega_{n}, x_{n}\right)_{n=-\infty}^{\infty}$ in $(\Sigma \times X)^{\mathbb{Z}}$ such that $x_{n+1}=\omega_{n}\left(x_{n}\right)$ is equipped with the induced product topology and the Borel $\sigma$-algebra $\mathcal{B}(\Omega)$. For our purposes, we may take $\Omega$ as the basic probability space 
and define the stochastic variables $X_{n}$ and $W_{n}, n \in \mathbb{Z}$, on $\Omega$ by $X_{n}(\boldsymbol{\omega}):=x_{n}$ and $W_{n}(\boldsymbol{\omega}):=\omega_{n}$ for $\boldsymbol{\omega}=\left(\omega_{n}, x_{n}\right)_{n=-\infty}^{\infty}$.

A stationary distribution $\mu$ of such a bi-infinite chain is shift invariant, i.e., $\mu=\mu \circ \mathcal{T}^{-1}$, where $\Omega \stackrel{\mathcal{T}}{\longrightarrow} \Omega$ is the usual right shift $(\mathcal{T} \boldsymbol{\omega})_{n}=\left(\omega_{n-1}, x_{n-1}\right)$. Moreover, the common marginal distribution $\mu \circ X_{n}^{-1}$ (any $n$ ) is invariant under $\mathcal{L}_{p}^{*}$. In fact, by the standard extension theorems, this marginal distribution determines $\mu$ uniquely. Dually, we can identify $f \circ X_{0}$ on $\Omega$ with a given function $f$ on $X$.

The distributions of such stationary Markov chains make up a convex closed set $\mathfrak{M}_{p}$ of Borel probability measures on $\Omega$ and we refer to them as $p$-measures. The set $\mathfrak{M}_{p}$ is partially ordered under absolute continuity $(\ll)$. In addition and this will be used below - the set $\mathfrak{M}_{p}$ is generated by (is the closed convex hull of) the minimal ergodic elements of this partial order. We say that the weighted IFS $(\mathfrak{S}, p)$ has the property of uniqueness of $p$-measures if $\mathfrak{M}_{p}$ has at most one element.

That the larger set $\mathfrak{M}(\mathcal{T})$ of $\mathcal{T}$-invariant measures is generated by its minimal elements is well known: see [9]. The set $\mathfrak{M}_{p}$ is moreover a down-set in $\mathfrak{M}(\mathcal{T})$, i.e., if $\tilde{\mu} \in \mathfrak{M}(\mathcal{T})$ and if $\tilde{\mu} \ll \mu \in \mathfrak{M}_{p}$, then $\tilde{\mu} \in \mathfrak{M}_{p}$ and it follows that $\mathfrak{M}_{p}$ must be generated by its own minimal elements. To show that $\mathfrak{M}_{p}$ is closed in this sense, let $f=d \tilde{\mu} / d \mu$ be the density of $\tilde{\mu}$ with respect to $\mu$ on $\mathcal{B}(X)$. The $\mathcal{T}$-invariance of $\tilde{\mu}$ and $\mu$ implies that $f=f \circ \mathcal{T} \bmod \mu$, so that $\left(\mathcal{L}_{p} h\right) f=\mathcal{L}_{p}(h f)$ $\bmod \mu$, for any $h \in C(X)$. Hence, we have that $\tilde{\mu} \in \mathfrak{M}_{p}$, since

$$
\int \mathcal{L}_{p} h d \tilde{\mu}=\int\left(\mathcal{L}_{p} h\right) f d \mu=\int \mathcal{L}_{p}(h f) d \mu=\int h f d \mu=\int h d \tilde{\mu} .
$$

\section{Main result}

Let $C_{a}^{b}(\boldsymbol{\omega})$, for $\boldsymbol{\omega} \in \Omega$ and $a<b \in \mathbb{Z}$, be the symbolic cylinder set of all elements $\tilde{\boldsymbol{\omega}}=\left(\tilde{\omega}_{i}, \tilde{x}_{i}\right)_{i \in \mathbb{Z}} \in \Omega$ such that $\tilde{\omega}_{i}=\omega_{i}$ for $i=a, a+1, \ldots, b$. Let $\mathcal{F}_{a}^{b}$ be the finite algebra generated by $\left\{\mathrm{C}_{a}^{b}(\boldsymbol{\omega}) \mid \boldsymbol{\omega} \in \Omega\right\}$.

For $n \geq 0$, we write $C_{n}(\boldsymbol{\omega})$ for the forward $n$-cylinder $C_{1}^{n}$ and $C_{-n}$ for the backward $n$-cylinder $\mathrm{C}_{-n+1}^{0}$. Since forward and backward cylinders are the ones we will consider, we will, for $n \geq 0$, lighten up the notation by replacing the sub- and super-scripts with subscripts $n$ and $-n$. Thus, we write $\mathcal{F}_{n}$ and $\mathcal{F}_{-n}$ for $\mathcal{F}_{1}^{n}$ and $\mathcal{F}_{-n+1}^{0}$, respectively.

For $a, b \in \mathbb{Z}$ and $\boldsymbol{\omega}=\left(\omega_{n}, x_{n}\right)_{n=-\infty}^{\infty} \in \Omega$, let $\Delta_{a}^{b} \subset X$ be the elementary image

$$
\Delta_{a}^{b}(\boldsymbol{\omega}):=\omega_{b} \omega_{b-1} \cdots \omega_{a}(X), \quad a \leq b,
$$

and $\Delta_{a}^{b}=X$ for $a>b$. Write, for $n \geq 0, \Delta_{n}$ for $\Delta_{1}^{n}$ and $\Delta_{-n}$ for $\Delta_{-n+1}^{0}$. Note that $x_{1} \in \Delta_{-n}(\boldsymbol{\omega})$ and $x_{n} \in \Delta_{n-1}(\boldsymbol{\omega})$. 
For $n \geq 0$, the $n$ :th variation of a function $p: \Sigma \times X \rightarrow \mathbb{R}$ is the positive function

$$
\operatorname{var}_{n} p(\boldsymbol{\omega}):=\sup _{x, y \in \Delta_{n-1}}\left|p\left(\omega_{n}, x\right)-p\left(\omega_{n}, y\right)\right|, \quad \text { for } n \geq 1,
$$

and $\operatorname{var}_{0} p:=\sup p-\inf p$. Note that $\operatorname{var}_{n} p$ is $\mathcal{F}_{n}$-measurable for all $n \geq 0$. In other words, $\left(\operatorname{var}_{n} p\right)_{n \geq 0}$ is a process adapted to the filtration $\left(\mathcal{F}_{n}\right)_{n \geq 0}$.

Theorem 3.1. Assume that $\inf p>0$ and assume that there is a $\tilde{\mu} \in \mathfrak{M}_{p}$ such that $\lim _{n \rightarrow \infty} \operatorname{diam} \Delta_{-n}=0, \tilde{\mu}$-almost surely. Suppose that $\left(R_{n}\right)_{n=0}^{\infty}$ is a real stochastic sequence adapted to $\left(\mathcal{F}_{n}\right)_{n=0}^{\infty}$, such that

$$
\sum_{n=0}^{\infty} R_{n}^{2} \in L^{1}(\tilde{\mu}),
$$

and suppose that

$$
\sum_{n} \tilde{\mu}\left\{\operatorname{var}_{n} p>R_{n}\right\}<\infty .
$$

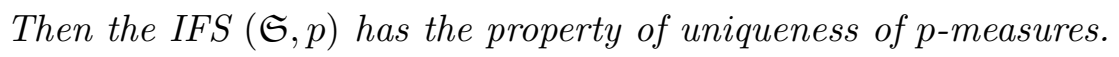

To see how Theorem 3.1 relates to Theorem 1.1: For a "well-behaved" covering transformation $T$ on $X$, we associate the IFS having the inverse branches of $T$ as its continuous maps (this may entail some minor modification of the space). The corresponding probabilistic weights are $p(\sigma, x):=g(\sigma x)$ for the given $g$-function.

In the IFS formalism above we used the variations $\operatorname{var}_{n} p, n \geq 1$, over forward cylinders. In Theorem 1.1 we used backward cylinders to define the variations $\operatorname{var}_{n} g(x), n \geq 1$, of the $g$-function, since the $n$th inverse image $I_{-n}(x)$ is the a.s. unique elementary image $\Delta_{-n}(\boldsymbol{\omega})$ containing $x$. Thus, $\operatorname{var}_{n} g\left(x_{1}\right)$ equals $\operatorname{var}_{n} p\left(\mathcal{T}^{n} \boldsymbol{\omega}\right)$. But this discrepancy is of no importance, since by the assumption that $\tilde{\mu}$ is $\mathcal{T}$-invariant, the conditions on $\operatorname{var}_{n} p$ in Theorem 3.1 are also $\mathcal{T}$-invariant, so that we have

$$
\tilde{\mu}\left\{\operatorname{var}_{n} p \circ \mathcal{T}^{n}>R_{n} \circ \mathcal{T}^{n}\right\}=\tilde{\mu}\left\{\operatorname{var}_{n} p>R_{n}\right\} .
$$

\section{The proof of Theorem 3.1 and the martingale $M_{n}$}

We assume that the measure $\tilde{\mu} \in \mathfrak{M}_{p}$ satisfies the conditions of the theorem. Let $\mu$ denote another $p$-measure. The purpose is to prove that $\tilde{\mu}$ is absolutely continuous with respect to $\mu$; hence there cannot be more than one ergodic $p$-measure and uniqueness follows. In Lemma 4.1 below, we prove absolute continuity of $\tilde{\mu}$ with respect to $\mu$, under the condition that the likelihood ratio martingale converge in $L_{1}(\mu)$. The convergence is then shown in the subsequent subsection.

Define, for $n \geq 0$, the process $M_{n}: \Omega \rightarrow \mathbb{R}_{+}$by

$$
M_{n}:=\frac{\tilde{\mu}\left\{\mathrm{C}_{n}\right\}}{\mu\left\{\mathrm{C}_{n}\right\}} .
$$


From the positivity condition inf $p>0$ follows that the $M_{n}$ 's are finite, i.e.,

$$
M_{n}(\boldsymbol{\omega})<\infty \text { for all } n \geq 0 \text { and } \boldsymbol{\omega} \in \Omega .
$$

It is now easy to see that the process $\left\{M_{n}, n \geq 0\right\}$ is a martingale (a likelihood ratio martingale) relative the filtration $\left(\mathcal{F}_{n}\right), n \geq 0$ and the probability $\mu$. Indeed, from (4.2) and the definition of $M_{n}$, we deduce that if $0 \leq m \leq n$ and $A \in \mathcal{F}_{m}$ then

$$
\int_{A} M_{n} d \mu=\sum_{\mathrm{C}_{n} \subset A} \frac{\tilde{\mu}\left\{\mathrm{C}_{n}\right\}}{\mu\left\{\mathrm{C}_{n}\right\}} \cdot \mu\left\{\mathrm{C}_{n}\right\}=\sum_{\mathrm{C}_{n} \subset A} \tilde{\mu}\left\{\mathrm{C}_{n}\right\}=\tilde{\mu}\{A\} .
$$

In the sum over the $C_{n}$, it is understood that we traverse a system of distinct representatives of the cylinders.

Recall that a process $\left\{U_{n}\right\}$ is uniformly integrable, $U I$, with respect to $\mu$ if

$$
\lim _{K \rightarrow \infty} \sup _{n} \int_{\left|U_{n}\right|>K}\left|U_{n}\right| d \mu=0 .
$$

From (4.3) above, we note that $\left\{M_{n}\right\}$ is $U I$ relative $\mu$ if it is tight relative $\tilde{\mu}$, i.e., if

$$
\sup _{n \geq 0} \tilde{\mu}\left\{M_{n}>K\right\} \rightarrow 0 \quad \text { as } K \rightarrow \infty .
$$

We only need this one-sided notion of tightness since $M_{n}>0$ and "tight" will henceforth refer to the property defined in (4.5). Note that $M_{n}$ is tight if and only if $\log M_{n}$ is tight (in this sense) and that a process $U_{n}$ is tight if it is bounded in $L_{p}(\tilde{\mu})$, i.e., if $\sup _{n} \int\left|U_{n}\right|^{p}<\infty$.

The Kakutani martingale approach of the paper is now formulated in the following lemma.

Lemma 4.1. If diam $\Delta_{-n} \rightarrow 0, \tilde{\mu}$ a.s. and the family $\left\{M_{n}: n \geq 0\right\}$, defined above, satisfies (4.5), then $\tilde{\mu} \ll \mu$.

Proof of Lemma 4.1. There is a well-known theorem (see [17, Ch. 14]) saying that if $\left(M_{n}\right)_{n=0}^{\infty}$ is a uniformly integrable $\mu$-martingale, then $M_{n} \rightarrow M_{\infty}$ in $L_{1}(\Omega, \mathcal{B}, \mu)$ as $n \rightarrow \infty$, where $M_{\infty}$ is non-negative and measurable with respect to the $\sigma$-algebra generated by $\cup_{n} \mathcal{F}_{n \geq 0}$. Furthermore, $M_{\infty}$ closes $M_{n}$, i.e., $M_{n}=$ $\mu\left[M_{\infty} \mid \mathcal{F}_{n}\right]$. Hence, on account of (4.3), we obtain

$$
\int_{A} M_{\infty} d \mu=\tilde{\mu}\{A\}
$$

for all $A \in \cup_{n>0} \mathcal{F}_{n}$.

By the ergodic theorem there is an $f \in L_{1}(\Omega, \mathcal{B}, \mu)$ such that

$$
\lim _{N \rightarrow \infty} \frac{1}{N} \sum_{k=0}^{N} M_{\infty} \circ \mathcal{T}^{k}=f
$$


with convergence in $L_{1}$. Thus, if $A \in \mathcal{F}_{a}^{b}$ is an arbitrary symbolic cylinder set then

$$
\int_{A} f d \mu=\lim _{N \rightarrow \infty} \frac{1}{N} \sum_{k=0}^{N} \int_{A} M_{\infty} \circ \mathcal{T}^{k} d \mu=\tilde{\mu}\{A\},
$$

since, by (4.6), $\int_{A} M_{\infty} \circ \mathcal{T}^{k} d \mu=\tilde{\mu}\{A\}$, for all large enough $k$, i.e., $k \geq-a+1$. This means that the measures $\tilde{\mu}$ and $f \mu$ will coincide on all symbolic cylinder sets.

Let $G$ be an arbitrary open set in $X$ and consider the open set $\Gamma:=X_{1}^{-1}(G) \subset$ $\Omega$. (Recall the definition of $X_{n}^{-1}$ from Section 2.) It is enough to show that $\tilde{\mu}(\Gamma)=(f \mu)(\Gamma)$, since this implies that $\tilde{\mu} \circ X_{1}^{-1}$ and $(f \mu) \circ X_{1}^{-1}$ are equal up to completions on the Borel $\sigma$-algebra of $X$. Hence, by the uniqueness of $\tilde{\mu}$ relative $\tilde{\mu} \circ X_{1}^{-1}$, we obtain that $\tilde{\mu}=f \mu$.

It remains to show that $\tilde{\mu}(\Gamma)=(f \mu)(\Gamma)$. Let $\Gamma_{1}$ be the set of $\boldsymbol{\omega} \in \Gamma$ such that $X_{1}\left(C_{-N}(\boldsymbol{\omega})\right) \subset G$ for some finite $N(\boldsymbol{\omega})$, i.e. $\Gamma_{1}$ is the union of all symbolic cylinders in $\cup_{n} \mathcal{F}_{-n}$ such that the corresponding elementary image is contained in $G$. Since $\tilde{\mu}$ and $f \mu$ coincide on cylinders, we have that $\tilde{\mu}\left(\Gamma_{1}\right)=(f \mu)\left(\Gamma_{1}\right)$, so it is enough to show that $\tilde{\mu}\left(\Gamma \backslash \Gamma_{1}\right)=(f \mu)\left(\Gamma \backslash \Gamma_{1}\right)$.

Note that, we have diam $\Delta_{-n} \rightarrow 0,(f \mu)$-almost surely as well as $\tilde{\mu}$-almost surely, since the event is contained in the $\sigma$-algebra generated by the cylinders in $\cup_{n} \mathcal{F}_{-n}$. But, for an element $\boldsymbol{\omega}$ of $\Gamma \backslash \Gamma_{1}$, we must have, for all $n \geq 0$

$$
\operatorname{diam} \Delta_{-n}(\boldsymbol{\omega}) \geq \operatorname{dist}\left(X_{1}(\boldsymbol{\omega}), X \backslash G\right)>0,
$$

since otherwise

$$
\inf _{\tilde{\boldsymbol{\omega}} \in \mathrm{C}_{-n}(\boldsymbol{\omega})} \operatorname{dist}\left(X_{1}(\tilde{\boldsymbol{\omega}}), X \backslash G\right)>0 .
$$

Thus, diam $\Delta_{-n}(\boldsymbol{\omega}) \nrightarrow 0$ for $\boldsymbol{\omega} \in \Gamma \backslash \Gamma_{1}$ and it follows that $\tilde{\mu}\left(\Gamma \backslash \Gamma_{1}\right)=(f \mu)(\Gamma \backslash$ $\left.\Gamma_{1}\right)=0$.

Remark 4. The second half of the proof of Lemma 4.1 is not necessary if, e.g., we are restricted to a one-sided shift dynamics on a symbol space.

4.1. Proving tightness. To complete the proof of Theorem 3.1, it remains to verify the tightness property (4.5) of $M_{n}$, where the $\mu$-martingale $M_{n}$ is constructed as in (4.1) from $\tilde{\mu}$ and any other $\mu \in \mathfrak{M}_{p}$.

Let $P_{n}(\boldsymbol{\omega}):=\mu\left\{\mathrm{C}_{n}(\boldsymbol{\omega}) \mid \mathrm{C}_{n-1}(\boldsymbol{\omega})\right\}$ and let $\tilde{P}$ be defined analogously. Thus, $M_{n}=\tilde{\mu}\left\{\mathrm{C}_{n}\right\} / \mu\left\{\mathrm{C}_{n}\right\}$ may be written

$$
M_{n}=\frac{\tilde{P}_{n} \cdots \tilde{P}_{1}}{P_{n} \cdots P_{1}}
$$

Note that $P_{n}(\boldsymbol{\omega})=\int p\left(\omega_{n}, x_{n}\right) d \mu_{n}\left(x_{n}\right)$ where $\mu_{n}$ is the distribution of $X_{n}$ conditioned on $\mathrm{C}_{n-1}$. Thus it is an integrated mean of $p\left(\omega_{n}, \cdot\right)$ over the elementary image $\Delta_{n-1}$. It follows that $\left|\tilde{P}_{n}-P_{n}\right| \leq \operatorname{var}_{n} p$ and that inf $p \leq P_{n}$. Moreover, $\sum_{\mathrm{C}_{n} \subset \mathrm{C}_{n-1}} P_{n}=1$ where the sum is understood to be taken for one representative of each cylinder. 
Taking the logarithm in (4.7) and using that $\log (1+x) \leq x$ we obtain, for any fixed positive integer $m_{0}$, that (with $n \wedge m:=\min \{n, m\}$ )

$$
\begin{aligned}
\log M_{n}= & \log M_{n \wedge m_{0}}+\sum_{i=m_{0}+1}^{n} \log \left(\tilde{P}_{i} / P_{i}\right) \\
& \leq \log M_{n \wedge m_{0}}+\sum_{i=m_{0}+1}^{n} \frac{\tilde{P}_{i}-P_{i}}{P_{i}} .
\end{aligned}
$$

Note that

$$
\frac{\tilde{P}_{i}-P_{i}}{P_{i}}=\frac{\tilde{P}_{i}-P_{i}}{\tilde{P}_{i}}+\frac{\left(\tilde{P}_{i}-P_{i}\right)^{2}}{\tilde{P}_{i} P_{i}} \leq \frac{\tilde{P}_{i}-P_{i}}{\tilde{P}_{i}}+\left(\tilde{P}_{i}-P_{i}\right)^{2} /(\inf p)^{2} .
$$

Hence, we see that

$$
\log M_{n} \leq \log M_{m_{0} \wedge n}+Z_{n}+(1 / \inf p)^{2} \cdot \sum_{i=m_{0}+1}^{n}\left(\tilde{P}_{i}-P_{i}\right)^{2} .
$$

where

$$
Z_{n}:=\sum_{i=m_{0}+1}^{n} \frac{\tilde{P}_{i}-P_{i}}{\tilde{P}_{i}}
$$

Note that $\log M_{m_{0} \wedge n}$ is bounded on account of (4.2).

Let $\left(R_{n}\right)_{n=0}^{\infty}$ be the adapted sequence satisfying the conditions of Theorem 3.1. Let $\epsilon>0$ be given and let $m_{0}=m_{0}(\epsilon)$ be the smallest $m \geq 1$ such that

$$
\sum_{n=m+1}^{\infty} \tilde{\mu}\left\{\operatorname{var}_{n} p>R_{n}\right\}<\epsilon .
$$

Furthermore, define $\tau=\tau(\boldsymbol{\omega})$ by

$$
\tau:=\inf \left\{n>m_{0}:\left|\tilde{P}_{n}-P_{n}\right|>R_{n}\right\}
$$

with the convention that $\inf \emptyset=\infty$. By the definition of $m_{0}$ we see that

$$
\tilde{\mu}\{\tau<\infty\}<\epsilon .
$$

Hence, since $\epsilon$ was chosen arbitrarily, it is clearly enough to show that $M_{n \wedge \tau}$ is $\tilde{\mu}$-tight.

From (4.8) we now see that, for all $n$,

$$
\log M_{n \wedge \tau} \leq \log M_{n \wedge m_{0}}+Z_{n \wedge \tau}+\frac{1}{(\inf p)^{2}} \sum_{i=m_{0}+1}^{n \wedge \tau}\left(\tilde{P}_{i}-P_{i}\right)^{2} .
$$

Moreover, for $m_{0}<i \leq n \wedge \tau$, we have $\left|\tilde{P}_{i}-P_{i}\right| \leq R_{i}$ Thus the $\tilde{\mu}$-integral of the last sum of (4.10) less than $\int \sum_{i=m_{0}+1}^{\infty} R_{i}^{2} d \tilde{\mu}<\infty$ and $M_{n \wedge \tau}$ is therefore $\tilde{\mu}$-tight if $Z_{n \wedge \tau}$ is tight. 
We observe that $Z_{n}$ is a $\tilde{\mu}$-martingale, since

$$
\begin{array}{r}
\tilde{\mu}\left[Z_{n}-Z_{n-1} \mid \mathcal{F}_{n-1}\right]=\sum_{\mathrm{C}_{n} \subset \mathrm{C}_{n-1}} \frac{\tilde{P}_{n}-P_{n}}{\tilde{P}_{n}} \cdot \tilde{P}_{n} \\
=\sum_{\mathrm{C}_{n} \subset \mathrm{C}_{n-1}}\left(\tilde{P}_{n}-P_{n}\right)=1-1=0 .
\end{array}
$$

The martingale difference sequence $Z_{i}-Z_{i-1}$ is equal to $\left(\tilde{P}_{i}-P_{i}\right) / \tilde{P}_{i}$ for $i \geq$ $m_{0}+1$ and is zero otherwise. Consequently we can use the Pythagorean property to obtain

$$
\int Z_{n}^{2} d \tilde{\mu}=\int \sum_{i=m_{0}+1}^{n}\left(\frac{\tilde{P}_{i}-P_{i}}{\tilde{P}_{i}}\right)^{2} d \tilde{\mu}
$$

and hence

$$
\int Z_{n}^{2} d \tilde{\mu} \leq \frac{1}{(\inf p)^{2}} \cdot \int \sum_{i=m_{0}+1}^{n}\left(\tilde{P}_{i}-P_{i}\right)^{2} d \tilde{\mu} .
$$

Note that $\tau$, defined in (4.9), is a stopping time relative $\left(\mathcal{F}_{n}\right)$ since both $\left(\operatorname{var}_{n} p\right)$ and $\left(R_{n}\right)$ are adapted to $\left(\mathcal{F}_{n}\right)$. Therefore, $Z_{n \wedge \tau}$ is also a $\tilde{\mu}$-martingale and we can use the estimate (4.11). We find that $Z_{n \wedge \tau}$ is bounded in $L_{2}(\tilde{\mu})$, since

$$
\int Z_{n \wedge \tau}^{2} d \tilde{\mu} \leq(1 / \inf p)^{2} \cdot \int \sum_{i=m_{0}+1}^{\infty} R_{i}^{2} d \tilde{\mu}<\infty .
$$

\section{The case with general weights}

For general weights $q$, i.e., weights not necessarily summing to 1 pointwise, we may define a set $\mathfrak{M}_{q}$ of invariant measures - q-measures — as positive eigenmeasures belonging to the maximum eigenvalue $\lambda$ of the adjoint to the positive operator $\mathcal{L}_{q}$. There is a well-known theory of translation from the case of general weights $q$ to the case of probabilistic weights $p$. The essential ingredient is the existence of a strictly positive eigen-function $h$ to the operator $\mathcal{L}_{q}$ which makes it possible to use the probabilistic weights

$$
p(\sigma, x)=\frac{1}{\lambda} q(\sigma, x) \cdot h(\sigma x) / h(x) .
$$

Then there is a bijective correspondence between $q$-measures $\nu$ and $p$-measures $\mu$ given by $\mathfrak{M}_{q} \ni \nu \stackrel{1-1}{\longrightarrow} \mu=h \nu \in \mathfrak{M}_{p}$.

In order to apply Theorem 3.1 to the general case, one must then somehow compute the variations $\operatorname{var}_{n} h(\sigma x) / h(x)$ as well as those of $q$. But, the construction of $h$ will many times be carried out in a highly non-constructive way by invoking various compactness theorems like the Arzela-Ascoli theorem. Proving square summability of variations of $h$ can then be a very hard task. 
We give therefore an alternative uniqueness result that might have applicability in such situations. We start from probabilistic weights given by general weights as in (5.1) for an a priori given eigen-function $h$.

Theorem 5.1. Let the weights of a probabilistic $\operatorname{IFS}(\mathfrak{S}, p)$ be given from a factorization as in (5.1) where $\inf q>0, \sup q<\infty$. Assume that there is a $\tilde{\mu} \in$ $\mathfrak{M}_{p}$ such that $\lim _{n \rightarrow \infty} \operatorname{diam} \Delta_{-n}=0 \tilde{\mu}$-almost surely and such that $\left\{\operatorname{var}_{n} \log h\right\}$ is $\tilde{\mu}$-tight. If $\left(R_{n}\right)_{0}^{\infty}$ is a random sequence satisfying

$$
\sum_{n=0}^{\infty} R_{n} \in L^{1}(\tilde{\mu}),
$$

such that

$$
\sum_{n} \tilde{\mu}\left\{\operatorname{var}_{n} q>R_{n}\right\}<\infty
$$

then the IFS (ㄷ, $p)$ has a unique p-measure.

Proof of Theorem 5.1. The argument for Theorem 5.1 is largely similar to that for Theorem 3.1 and we just make a sketch of it here. Let $\nu$ and $\tilde{\nu}$ be the corresponding measures in $\mathfrak{M}_{q}$, i.e., $h \nu=\mu$ and $h \tilde{\nu}=\tilde{\mu}$ where $\tilde{\mu}$ satisfies the conditions of the theorem. By adjusting the weights $q=q / \lambda$ if necessary, we may assume that $\lambda=1$ in (5.1). The construction of $M_{n}$ and Lemma 4.1 will work in exactly the same way.

The argument for verifying (4.5) is similar but not identical to that above: Note that the factorization (5.1) implies that

$$
\begin{aligned}
\mu\left(\mathrm{C}_{n}\right) & =\int p\left(\omega_{n}, x_{n}\right) \cdots p\left(\omega_{1}, x_{1}\right) d \mu\left(x_{1}\right) \\
& =\int h\left(x_{n}\right) q\left(\omega_{n}, x_{n}\right) \cdots q\left(\omega_{1}, x_{1}\right) d \nu\left(x_{1}\right)
\end{aligned}
$$

due to the fact that the product of the factors $h\left(x_{i-1}\right) / h\left(x_{i}\right)$ telescopes in the integrand and $\frac{d \mu\left(x_{1}\right)}{h\left(x_{1}\right)}=d \nu\left(x_{1}\right)$. Hence, instead of the factorization (4.7), we obtain that $M_{n}=\mu\left\{\mathrm{C}_{n}\right\} / \tilde{\mu}\left\{\mathrm{C}_{n}\right\}$ is given by

$$
M_{n}=\frac{\tilde{h}_{n}}{h_{n}} \cdot \frac{\tilde{Q}_{n} \cdots \tilde{Q}_{1}}{Q_{n} \cdots Q_{1}}
$$

where for $n \geq 1$

$$
\begin{aligned}
Q_{n} & =\int q\left(\omega_{n}, x_{n}\right) d \nu_{n-1}(x) \\
h_{n} & =\int h\left(x_{n}\right) d \nu_{n}\left(x_{n}\right) \\
d \nu_{n} & =\prod_{j=1}^{n} q\left(\omega_{j}, x_{j}\right) d \nu\left(x_{1}\right) / \int \prod_{j=1}^{n} q\left(\omega_{j}, x_{j}\right) d \nu\left(x_{1}\right) .
\end{aligned}
$$


and the quantities $\tilde{Q}_{n}, \tilde{h}_{n}$ and $\tilde{\nu}_{n}$ are analogously defined. Note that, $Q_{n}$ is again a weighted average of the weights over the elementary image $\Delta_{n-1}$.

Corresponding to (4.8) we obtain

$$
\log M_{n} \leq \log M_{m_{0}}+\left|\log \tilde{h}_{n}-\log h_{n}\right|+\left|\log \tilde{h}_{m_{0}}-\log h_{m_{0}}\right|+Z_{n}
$$

where (with $m_{0}$ playing the same role as in the proof of Theorem 3.1)

$$
Z_{n}:=\sum_{i=m_{0}+1}^{n} \frac{\tilde{Q}_{i}-Q_{i}}{Q_{i}} .
$$

Note that $\left|\log \tilde{h}_{n}-\log h_{n}\right| \leq \operatorname{var}_{n} \log h$ is $\tilde{\mu}$-tight by assumption. (But the process $\left\{Z_{n}\right\}$ is not a $\tilde{\mu}$-martingale in this case.)

Introducing a random time $\tau$ as in Theorem 3.1 and noting that

$$
\int Z_{n \wedge \tau} d \tilde{\mu} \leq \text { const. } \cdot \int \sum_{i=m_{0}+1}^{\infty} R_{i} d \tilde{\mu}<\infty,
$$

and the result follows; we omit the details.

Acknowledgements. AÖ was supported in part by Institut Mittag-Leffler and both authors acknowledge the hospitality of the institute. We would like to thank the anonymous referee for many valuable suggestions. In addition, many thanks to Svante Janson, Yuval Peres, Anthony Quas, Jeff Steif and Örjan Stenflo for pointing out errors and suggesting improvements of earlier versions of this paper.

\section{References}

[1] M. Aizenman, J. Chayes, L. Chayes and C. Newman, Discontinuity of the magnetization in the one-dimensional $1 /|x-y|^{2}$ Ising and Potts models, J. Statist. Phys. 50 (1988), $1-40$.

[2] H. Berbee, Chains with Infinite Connections: Uniqueness and Markov Representation, Probab. Theory Related Fields 76 (1987), 243-253.

[3] M. Bramson and S. Kalikow, Nonuniqueness in g-functions, Israel J. Math. 84 (1993), 153-160.

[4] W. Doeblin and R. Fortet, Sur les châines á liasons complètes, Bull. Soc. Math. France 65 (1937), 132-148.

[5] A. Fan and Y. Jiang, On the Ruelle-Perron-Frobenius Operators. I. Ruelle Theorem, Comm. Math. Phys. 223 (2001), 125-141.

[6] A. Fan and K.-S. Lau, Iterated function system and Ruelle operator., J. Math. Anal. Appl. 231 (1999), 319-344.

[7] T.E. Harris, On chains of infinite order, Pacific J. Math. 5 (1955), 707-724.

[8] M. Keane, Strongly Mixing g-Measures, Invent. Math. 16 (1972), 309-324.

[9] G. Keller, Equilibrium States in Ergodic Theory, L M S Student Texts 42, Cambridge University Press 1998.

[10] _ Generalized Bounded Variation and Applications to Piecewise Monotonic Transformations, Z. Wahrscheinlichkeitstheorie verw. Gebiete 69 (1985), 461-478.

[11] K.-S. Lau and Y.-L. Ye, Ruelle operator with nonexpansive IFS, Studia Math. 148 (2001), 143-169.

[12] M. Pollicott, Rates of mixing for potentials of summable variation, Trans. Amer. Math. Soc. 352 (2000), 843-853. 
[13] A. Quas, Non-ergodicity for $C^{1}$ expanding maps and $g$-measures, Ergodic Theory Dynam. Systems 16 (1996), 531-543.

[14] Ö. Stenflo, Uniqueness of invariant measures for place-dependent random iterations of functions, Survey, IMA Vol. Math. Appl. 132 (2002), 13-32.

[15] P. Walters, Ruelle's operator theorem and g-measures, Trans. Amer. Math. Soc. 214 (1975), 375-387.

[16] _ Invariant measures and equilibrium states for some mappings which expand distances, Trans. Amer. Math. Soc. 236 (1978), 121-153.

[17] D. Williams, Probability with Martingales, Cambridge University Press, 1991.

Anders Johansson, Division of Mathematics and Statistics, University of Gävle, SE-801 76 GÄVLE, SWEDEN

E-mail address: ajj@hig.se

Anders Öberg, Department of Mathematics, Uppsala University, P.O. Box 480, SE-751 06 UPPSALA, SWEDEN

E-mail address: anders@math.uu.se 